\title{
Proposal for revision of the European Laryngological Society classification of endoscopic cordectomies
}

\author{
Marc Remacle - Christophe Van Haverbeke • Hans Eckel • Patrick Bradley • \\ Dominique Chevalier · Votko Djukic • Marco de Vicentiis • Gerhard Friedrich • \\ Jan Olofsson · Giorgio Peretti · Miquel Quer · Jochen Werner
}

Published online: 13 April 2007

(C) Springer-Verlag 2007

\section{Erratum to: Eur Arch Otorhinolaryngol 264:499-504 DOI 10.1007/s00405-007-0279-z}

Owing to an unfortunate oversight, an important proof correction was not carried out. Wrong addresses were given for J. Olofsson and G. Peretti.

The correct addresses are given below:

J. Olofsson

Department of Oto-rhino-laryngology and Head \& Neck Surgery, University Hospital of Bergen, Bergen, Norway
The online version of the original article can be found at http://dx.doi.org/10.1007/s00405-007-0279-z.

M. Remacle $(\bowtie) \cdot$ C. Van Haverbeke $\cdot$ M. Quer $\cdot$ J. Werner

Department of Oto-rhino-laryngology and Head \& Neck Surgery, University Hospital of Louvain at Mont-Godinne,

Dr G. Therasse Avenue, 1, 5530 Yvoir, Belgium

e-mail: remacle@ orlo.ucl.ac.be

H. Eckel

Department of Oto-rhino-laryngology and Head \& Neck Surgery, Regional Hospital, Klagenfurt, Austria

\section{P. Bradley}

Department of Oto-rhino-laryngology and Head \& Neck Surgery, Queens Medical Centre, Nottingham, NG7 5EU, UK

\section{Chevalier}

Department of Oto-rhino-laryngology and Head \& Neck Surgery, Hospital Claude Huriez, University of Lille, Lille, France

\section{Djukic}

Department of Oto-rhino-laryngology and Head \& Neck Surgery Medical Center,

University of Belgrade, Belgrade, Yugoslavia

\section{G. Peretti}

Department of Oto-rhino-laryngology and Head \& Neck Surgery, University Hospital of Brescia, Brescia, Italy

We apologize for any inconvenience caused.

M. de Vicentiis

Hospital La Sapienza, University of Rome, Rome, Italy

G. Friedrich

Department of Oto-rhino-laryngology and Head \& Neck Surgery,

University Hospital of Graz, Graz, Austria

J. Olofsson

Department of Oto-rhino-laryngology and Head \& Neck Surgery,

University Hospital of Bergen, Bergen, Norway

G. Peretti

Department of Oto-rhino-laryngology and Head \& Neck Surgery,

University Hospital of Brescia, Brescia, Italy

M. Quer

Department of Oto-rhino-laryngology and Head \& Neck Surgery,

Hospital Sant Pau, Barcelona, Spain

J. Werner

Department of Oto-rhino-laryngology and Head \& Neck Surgery,

University Hospital of Marburg, Marburg, Germany 